\title{
Potential Protection of Vitamin C and Bungur Leaf against Ulcerative Colitis
}

\author{
Sabilla Amrina Rosyada ${ }^{1}$, Meddy Setiawan ${ }^{2}$, Rahayu $^{3}$ \\ ${ }^{1}$ Medical Student at Faculty of Medicine, Muhammadiyah Malang University, Malang, East Java, \\ Indonesia, 65144 \\ ${ }^{2}$ Lecturer and Internist at Faculty of Medicine, Muhammadiyah Malang University, Malang, East Java, \\ Indonesia, 65144 \\ ${ }^{3}$ Lecturer and Neurologist at Faculty of Medicine, Muhammadiyah Malang University, Malang, East Java, \\ Indonesia, 65144 \\ Corresponding Author: Sabilla Amrina Rosyada
}

DOI: https://doi.org/10.52403/ijrr.20220172

\begin{abstract}
Background/Aim: Ulcerative colitis is an inflammation of the large intestine with symptoms such as abdominal pain, bloody diarrhea, and rectal urgency. Ulcerative ix colitis is the most common form of inflammatory bowel disease worldwide (6-12 per 100,000 population with a prevalence of $76-$ 150 per 100,000 population). The exact etiology of ulcerative colitis is unknown. However, it is suspected that genetics, environmental factors, and microbiota play a role in the pathogenesis of ulcerative colitis. This study to review effect of Bungur leaves (Lagerstroemia speciosa) and vitamin $\mathrm{C}$ in improving the condition of ulcerative colitis.
\end{abstract}

Method: The used method in this article is a narrative review by collecting data from existing research, using 24 journals. 14 Q1 indexed journals, 7 Q2 indexed journals, 4 Q3 indexed journals, and 1 textbook. Based on the results of the research collected, the effect of vitamin $\mathrm{C}$ and extracts of bay leaf as anti-inflammatory in ulcerative colitis will be shown. The research is based on PICO (Population, Intervention, Comparation, Outcome), using the Journal search tool obtained from the Pubmed, Google Scholar, and NCBI search tools, which are used as research material and processed into new information related to the purpose of writing.

Results and Discussion: Based on the results of the literature review, vitamin $\mathrm{C}$ and Lagerstoemia $s$. leaves can play a role in overcoming oxidative stress in ulcerative colitis by reducing levels of proinflammatory cytokines.

Conclusion: Vitamin $\mathrm{C}$ and Bungur leaves play a role in improving the condition of ulcerative colitis.

Keywords: Anti-inflammation, Ulcerative colitis, Vitamin C, Lagerstroemia speciosa, Bungur Leaves

\section{INTRODUCTION}

Ulcerative colitis is inflammation of the colon, the symptoms of which are pain in the abdomen, hematochezia, and rectal urgency. The diagnosis of ulcerative colitis is made on the basis of clinical presentation and endoscopic evidence of inflammation of the colon beginning in the rectum and extending proximally in the colon. This disease is included in one of the chronic inflammatory bowel diseases, namely inflammatory bowel disease. The hallmark of this inflammation is that it is limited to the mucosa and submucosa of the large intestine.(1)

Ulcerative colitis is the most common form of inflammatory disease of the colon worldwide (6-12 per 100,000 population with a prevalence of 76-150 per 100,000 population). Geographical factors, high economics (often found in tropical and industrialized countries), immune factors and oxidative stress have an important role 
in ulcerative colitis. In the United States, the disease accounts for a quarter of a million doctor visits each year and the medical costs directly related to the disease are estimated to exceed 4 billion dollars per year. In Indonesia, there are between 5-10 cases per year in studies conducted at RSCM. By knowing the data above, it can be seen that from year to year the prevalence of ulcerative colitis is increasing.(2)

The prevalence of ulcerative colitis at Cipto Mangunkusumo Hospital, Jakarta in 1991-1995 reached $2.5 \%$. The incidence of this disease in women and men is generally equal, or sometimes slightly more in women. The age pattern of patients with this disease is bimodal, with peaks in the age range of 15-25 years and 55-65 years, although it can occur in various age groups. The exact etiology of ulcerative colitis is not known with certainty. Ulcerative colitis is closely related to human interactions with the environment (smoking, diet, use of nonsteroidal anti-inflammatory drugs), genetics and immunoregulatory factors. $(3,4)$

The first-line drug in ulcerative colitis is 5-aminosalicylic acid (5-ASA) with mild to moderate clinical severity in which reactive oxygen species (ROS) are involved in the metabolism of prostaglandins and leukotrienes and can act as second messengers, so ROS transport may be a single mechanism of action of 5ASA that exerts an anti-inflammatory effect. In addition to 5-ASA, corticosteroids are also often used in patients with ulcerative colitis with severe clinical severity. Although in its application synthetic drugs are still often found, alternative choices of other medicines such as traditional medicines, herbal plants, and medicinal plants are still the main choice for the majority of developing people because they are affordable and easy to obtain due to their abundant availability in nature.(5)

Leeks (Lagerstroemia speciosa) is known to have several biological activities which have been inferred from most of the research activities that are played by corosolic acid. In addition to corosolic acid, other phytoconstituents of curcuma leaf including gallotannin, and lagerstroemia can also contribute to the bioactivity of curcuma leaf. One example of the biological activity possessed by Lagerstroemia speciosa is as an anti-inflammatory which is played by ethyl acetate and ethanol. In previous studies, ethyl acetate was able to significantly reduce edema. $(6,7)$

Protective effect of leaf methanol extract Lagerstroemia speciosa evaluated for ulcerative colitis. Histology of the colonic sections revealed inflammatory changes and a marked decrease in the integrity of the mucosal layer with inflammatory changes. Both doses of currant leaf extract significantly prevented the inflammatory and ulcerative damage of the colon, reduced lipid peroxidation and also restored the levels of innate antioxidants in the colon tissue. These findings indicate a protective effect of curcas leaf extract against inflammatory and oxidative damage. $(8,9)$

Previous research has shown that vitamin $\mathrm{C}$ has a role as an immunostimulant and as an anti-inflammatory. Vitamin $\mathrm{C}$ inhibits microglia activation and proinflammatory cytokine production, including tumor necrosis factor- $\alpha$ (TNF- $\alpha$ ) and interleukin-1 $\beta(\mathrm{IL}-1 \beta)$. In addition, vitamin $\mathrm{C}$ plays a role in reducing malondialdehyde (MDA), increase activity superoxide dismutase (SOD), and modulates the ratio of $\mathrm{Bax} / \mathrm{Bcl}-2$ and p-p38 MAPK activation in the hippocampus. ulcerative. (10-12)

Broadly speaking, genetics, environmental factors, microbiota, and lifestyle influence the development of ulcerative colitis. In ulcerative colitis NF-kB phosphorylation occurs, where NF-B induces the expression of inflammatory mediators (cytokines, chemokines, TNFalpha, IL-1B, IL6) \& various immune responses. In addition, NF-kB also regulates the inflammatory protein response (COX-2 $\&$ iNOS) which ultimately causes oxidative stress. Host defense also decreases due to damage to the epithelial barrier which 
causes infiltration of inflammatory cells in the mucosa and submucosa of the colon which is called ulcerative colitis. Giving leaf extract Lagerstroemia speciosa and vitamin $\mathrm{C}$ proved to be able to improve the condition of ulcerative colitis. The content of methanolic, ethyl acetate, ethanol, and corosolic acid in Bungur leaves is able to work as an anti-inflammatory, and vitamin $\mathrm{C}$ reduces the potential for scarring through the mechanism of inhibiting cross-linking of collagen fibers and fibrosis which play an important role in the formation of new blood vessels (angiogenesis). In addition, vitamin $\mathrm{C}$ was also able to reduce the production of proinflammatory cytokines, TNF alpha, IL-1B, IL-6, IL-17, and mRNA. Vitamin $\mathrm{C}$ is also able to block the NF-kB pathway by inhibiting the production of cytokines and chemokines. $(9,13)$

\section{METHOD}

This article review based on the topic of discussion, the literature search is in the form of journals determined from 20162021 indexed by ISSN, national journals, and international journals. Writing is based on PICO (Population, Intervention, Comparation, Outcome) as follows: Population: Ulcerative colitis; Intervention: Colon ulcerative colitis; Comparison: Normal colon; Outcome: Ulcerative colitis improvement.

Inclusion criteria Research journals with research subjects in the form of ulcerative colitis, journals showing the role of vitamin $\mathrm{C}$ in inflammation/ulcerative colitis, journals showing the role of Bungur leaves and their active ingredients in inflammation/ulcerative colitis, journals published within the last 5 years. Inclusion criteria for journals in languages other than English and Indonesian and paid research journals.

\section{RESULT AND DISCUSSION}

Genetics plays a role in ulcerative colitis. $8 \%-14 \%$ of patients with ulcerative colitis (KU) have a family history of UC. In addition, someone with 1 st degree relatives is also 4x more at risk. More than 200 loci are associated with IBD, 30 of which are specific for KU, 41 for Chron's disease, 137 shared in both, where gene loci function as: autophagy, microbial recognition, lymphocyte and cytokine signaling.(4)

Genes in IBD regulate mucosal barrier integrity and function, innate immune responses, and microbial homeostasis. In ulcerative colitis there is a mutation in the CARD15 gene which causes an increase in the interaction between bacteria and the host defense system. The altered balance of the pathogenic microbiota creates a pro-inflammatory environment that exacerbates inflammation in the intestine. Disfunction of the immune system, both innate and adaptive, is caused by loss of tolerance to commensal bacteria, leading to an abnormal inflammatory response in the intestinal tract of patients with IBD. $(3,4,14)$

Dysfunction in goblet cell function is thought to be one of the causes of ulcerative colitis. Goblet cells synthesize and release mucin which functions as a physical barrier (protects from digestive enzymes and bacterial adhesion), and helps the movement and effective diffusion of colonic contents. (Amandip Kaur and Paraskevi). Environmental factors such as: pollution, diet (5 of 7 studies showed that red meat consumption was associated with the incidence of ulcerative colitis, 3 of 4 studies showed excessive seafood consumption was associated with KU, consumption of unsaturated fats, and n-6 fatty acids increased the risk of UC whereas a diet high in n-3 fatty acids reduces the risk of UC), and the use of antibiotics is associated with the incidence of KU.(4)

In microbiota factors, it is not known whether dysbiosis is the result or initiator of the occurrence of UC (in dysbiosis there is no SCFA production, so there is no availability of energy for the epithelium, mucus production, and colonic proliferation). In addition, a decrease in the number of Ruminococcaceae and Lachnospiraceae, followed by an increase in the number of Enterobacteriaceae and 
Fusobactericeae, which are prophylactic microbes, is also associated with the incidence of inflammation in ulcerative colitis. In ulcerative colitis there is a complex inflammation of innate and adaptive immune cells that infiltrates the lamina propria, resulting in an increase in neutrophils that transmigrate to the colonic epithelium and die (short-lived neutrophils).(15)

According to a study conducted by Kazimierz Wielki University, supplementation of $1000 \mathrm{mg}$ of vitamin C per day positively affected the immune response through the expression of IL-6, IL10, CCL 2, AND CRP. CCL 2 transports monocytes, memory $\mathrm{T}$ cells, and dendritic cells to induce a pro-inflammatory response caused by tissue damage. CRP is a nonspecific marker of ongoing inflammation, tissue damage, and necrosis. IL-1 and IL-6 are proinflammatory proteins (IL-6 is inhibited by the expression of IL-10 which is anti-inflammatory). Vitamin C supplementation is known to increase antioxidant activity and reduce oxidative stress which results in decreased expression of genes associated with inflammation due to oxidative stress induction. $(16,17)$

High doses of vitamin $\mathrm{C}(4 \mathrm{~g} / \mathrm{kg})$ in ulcerative colitis can reduce levels of IL-6, TNF-alpha, $\mathrm{H} 2 \mathrm{O} 2$, and increase levels of fibroblasts, type 1 collagen, type 3 collagen, catalase, and glutathione peroxidase. In addition, vitamin $\mathrm{C}$ was able to inhibit the expression of NF-kB so that COX-2 and iNOS levels decreased. Levels of MPO, MDA, and inflammatory cytoclines are known to decrease, resulting in an improvement in ulcerative colitis.(16)

Administration of vitamin $\mathrm{C}$ at a dose of $60 \mathrm{mg} / \mathrm{kg}$ reduces oxidative stress and inflammation by regulating proinflammatory cytokines (TNF alpha, ILIB, IL-6) in ulcerative colitis, increases DAI scores (increased body weight, hematochezia, and stool consistency), improves colon histology. One of the risk factors for inflammatory bowel disease is the dysfunction of the interaction between the colonic microbiota and the mucosal immune system. Administration of vitamin C $60 \mathrm{mg} / \mathrm{kg}$ has been shown to be able to reduce microbial dysbiosis. Vitamin $\mathrm{C}$ which is taken regularly at $120 \mathrm{mg} /$ day is known to prevent chronic disease.(18)

The content of vitamin $\mathrm{C}$ isolated from Lycium barbarum has been shown to reduce the effect on IBD as indicated by slowing weight loss, compaction of faeces, lowering the severity index of ulcerative colitis, blocking proinflammatory cytokines, increasing colon length, and modulating the composition of the colonic microbiota. $(12,19)$

The National Institute of Laser Enhanced Sciences conducted a study to investigate the role of vitamin $\mathrm{C}$ in inflammation. As a result, vitamin $\mathrm{C}$ is able to regulate the inflammatory process, stimulate tissue repair, modulate the release of catabolic cytokines that stimulate inflammation, and activate phagocytosis. In addition, vitamin $\mathrm{C}$ is known to decrease levels of TNF alpha, IL-IB but increase levels of superoxide dismutase. A study conducted by Cairo University showed that administration of vitamin $\mathrm{C}$ reduced edema and inflammatory cells. In addition, there is an increase in the number of fibroblasts that form collagen fibers that play a role in new tissue formation and angiogenesis.(20)

Universiti Putra Malaysia conducted a study on subjects treated with vitamin $\mathrm{C}$ $1000 \mathrm{mg} /$ day with the aim of identifying the role of vitamin $\mathrm{C}$ in lowering levels of markers of inflammation. From this study, it was found that vitamin $\mathrm{C}$ acts as an electron donor for enzymes in the human body and neutralizes free radicals to protect against oxidative stress. In addition, vitamin $\mathrm{C}$ also reduces high levels of cytokines and IL-6 (associated with reduced nitric oxide, increased ROS leading to endothelial and microvascular dysfunction). High levels of IL-6 stimulate the liver to synthesize and secrete the low-grade systemic inflammatory marker C-Reactive Protein (CRP). Lagerstroemia speciosa contains ethyl acetate, ethanol, and methanol which 
act as anti-oxidants (where ethyl acetate and ethanol have higher antioxidant content than methanol, and ethyl acetate reduces inflammation better than ethanol) whose mechanism of action is by inhibiting the cyclooxygenase enzyme. $(2,7,21)$

This is also supported by a study conducted by Chaudhari et al where both doses of LS extract containing corosolic acid, flavonoids, saponins, tannins, ethyl acetate, ethanol were shown to significantly prevent inflammatory and ulcerative damage to the intestines, reduce lipid peroxidation and also restore blood sugar levels. innate antioxidants in colon tissue. It is well known that oxidative stress damages cellular macromolecules such as DNA, lipids, and proteins which then induces ulcerative colitis. Leaf extract (Lagerstroemia speciosa) is known to be able to reduce MDA levels (in ulcerative colitis there is an increase in MDA due to lipid peroxidation). In addition, parsley leaf extract. It is also known to increase the activity of SOD and other antioxidant enzymes. The administration of curcuma leaf extract also reduces the entry of neutrophils into the lamina propria and submucosa (in ulcerative colitis there is an influx of neutrophils, monocytes, and macrophages into the lamina propria submucosa. In this study, it was concluded that the administration of LS significantly increased the activity of innate antioxidant enzymes. Research conducted by Khon Khaen University, Thailand revealed that the antioxidant activity between vitamin C and Lagerstroemia Speciosa the same size. In addition, the antioxidant effect between Lagerstroemia speciosa and L. floribunda were also tested. The result, antioxidant effect Lagerstroemia Speciosa greater than the antioxidant effect on L. floribunda. Ethanol content in Lagerstroemia speciosa has high antioxidant activity. Lagerstroemia speciosa also known to be able to inhibit the activity of MMP-2 and MMP-9 (responsible for collagen degradation).(11)

Research conducted by the University of Maringa revealed that ethyl acetate was able to prevent oxidative stress by reducing free radical levels within 24 hours and increasing tissue repair on the 7 th day of observation (on macroscopic and histological images). Keimyung University School of Medicine revealed that the corosolic acid content in Lagerstroemia speciosa plays a role in ameliorating acute inflammation by suppressing phosphorylation and transcription of IRAK1. This suggests the possibility of using corosolic acid in the treatment of acute inflammation. Inflammation is caused by several stimulants such as: infectious agents, environmental factors, antigen-antibody reactions, psychological and pathological factors, and free radicals. The content of ethyl acetate, ethanol, and methanol in the Lagerstroemia speciosa has leaves antioxidant activity that has been tested in studies of acute and chronic inflammatory diseases, as well as abdominal pain. The activity of ethyl acetate is known to be greater than the antioxidant activity of ethanol and methanol(21)

The content of flavonoids and saponins in Bungur leaves is known to have anti-inflammatory, anti-nociceptive, and antirheumatic activities (leaf extracts). Lagerstroemia speciosa can scavenge superoxide anions (superoxide participates in the formation and recruitment of polymorphonuclear (PMN)) thereby inhibiting inflammation.(11,21)

Vitamin $\mathrm{C}$ stimulates the migration of neutrophils to the site of infection, enhances phagocytosis, generation of antioxidants, kills microbes. Low-dose vitamin $\mathrm{C}$ supplementation (0.25-0.8g/day) reduced hospitalization rates by $19 \%$, while higher doses of vitamin $\mathrm{C}$ reduced hospitalization rates by $36 \%$. Prevention of inflammation can be done by consuming vitamin C (100-200mg/day). As for treatment efforts, higher doses (grams) are needed to compensate for the increased inflammatory response and metabolic needs.(19) Pretreatment using vitamin C inhibited the activation of microglia and the production of proinflammatory cytokines 
(TNF alpha and IL-IB), decreased levels of malondialdehyde, increased SOD so as to modulate oxidative stress and inflammatory response. (22)

According to a study conducted by the University of Medicine Berlin, administering vitamin $\mathrm{C}$ to healthy subjects increased NK cells, lymphocyte proliferation, and chemotaxis. High doses of vitamin $\mathrm{C}$ besides stimulating immune cells (especially dendritic cells, IL-12) also activates the function of $\mathrm{B}$ cells and $\mathrm{T}$ cells. In the GIT system, vitamin C plays an important role as a micronutrient and as an antioxidant in protecting intestinal cells from inflammatory stimuli. In patients with IBD such as ulcerative colitis, the concentration of vitamin $\mathrm{C}$ in the mucosa decreases drastically. High-dose injection of vitamin $\mathrm{C}$ has been shown to reduce morbidity in IBD patients. The body cannot synthesize vitamin $\mathrm{C}$ by itself. Most of the vitamin $\mathrm{C}$ is completely absorbed in the small intestine. The percentage of vitamin $\mathrm{C}$ absorbed decreases with increasing intraluminal concentration. Vitamin $\mathrm{C}$ acts as a powerful antioxidant in various reactions and metabolic processes, stabilizes vitamin $E$ and folic acid, increases the absorption of iron and toxins, and weakens the inflammatory response. $(16,17,23)$

\section{CONCLUSION}

Active constituent on Lagerstroemia speciosa as: ethyl acetate, methanolic, ethanol, corosolic acid, gallotannin, lagerstroemin, and ethanol has an important role as anti-inflammatory (significantly able to prevent inflammation in ulcerative colitis, reduce lipid peroxidation, and also restore the level of innate antioxidants in the colon tissue). Vitamin $\mathrm{C}$ has been shown to be able to improve the condition of ulcerative colitis by inhibiting the activation of the NF-kB pathway so that oxidative stress and inflammatory responses can be reduced. In addition, vitamin $\mathrm{C}$ is able to reduce the potency of scaring through the mechanism of inhibition of collagen fiber cross-linking and fibrosis, and is also important for the formation of new blood vessels (angiogenesis). Vitamin $\mathrm{C}$ acts as a cofactor in hydroxyproline synthesis to produce type IV collagen and enhances the vitality and function of endothelial cells.

\section{Funding}

This work did not receive any grant from funding agencies in the public, commercial, or not-for-profit sectors.

\section{Conflict of Interest}

There are no conflicts of interest to declare by any of the authors of this study.

\section{Acknowledgement: None}

Ethical Approval: Not Applicable

\section{REFERENCES}

1. Tripathi K, Feuerstein JD. New developments in ulcerative colitis: Latest evidence on management, treatment, and maintenance. Drugs Context. 2019;8:1-11.

2. Gajendran $M$, Loganathan $P$, Jimenez $G$, Catinella AP, Ng N, Umapathy C, et al. A comprehensive review and update on ulcerative colitis,. Disease-a-Month. 2019; 65(12).

3. Kaur A, Goggolidou P. Ulcerative colitis: Understanding its cellular pathology could provide insights into novel therapies. J Inflamm (United Kingdom). 2020;17(1):18.

4. Du L, Ha C. Epidemiology and Pathogenesis of Ulcerative Colitis. Gastroenterol Clin North Am. 2020; 49(4): 643-54.

5. Rubin DT, Ananthakrishnan AN, Siegel CA, Sauer BG, Long MD. ACG Clinical Guideline: Ulcerative Colitis in Adults. Am J Gastroenterol. 2019;114(3):384-413.

6. Kim SJ, Cha JY, Kang HS, Lee JH, Lee JY, Park JH, et al. Corosolic acid ameliorates acute inflammation through inhibition of IRAK-1 phosphorylation in macrophages. BMB Rep. 2016;49(5):276-81.

7. Thakur RS, Devaraj E. Lagerstroemia speciosa (L.) Pers. triggers oxidative stress mediated apoptosis via intrinsic mitochondrial pathway in HepG2 cells. Environ Toxicol. 2020;35(11):1225-33. 
8. Mousa AM, El-Sammad NM, Abdel-Halim AH, Anwar N, Khalil WKB, Nawwar M, et al. Lagerstroemia speciosa (L.) pers leaf extract attenuates lung tumorigenesis via alleviating oxidative stress, inflammation and apoptosis. Biomolecules. 2019;9(12):122.

9. AL-SNAFI AE. Medicinal Value of Lagerstroemia Speciosa: an Updated Review. Int J Curr Pharm Res. 2019;11(5): $18-26$.

10. Sauberlich HE. Ascorbic Acid (Vitamin C). Clin Lab Med. 1981;1(4):673-84.

11. Kolakul P, Sripanidkulchai B. Phytochemicals and anti-aging potentials of the extracts from Lagerstroemia speciosa and Lagerstroemia floribunda. Ind Crops Prod. 2017;109(September):707-16.

12. Rohit Singh T, Ezhilarasan D. Lagerstroemia speciosa (L.) Pers., ethanolic extract attenuates simultaneously administered isoniazid- and dapsoneinduced hepatotoxicity in rats. J Food Biochem. 2021;45(8):1-13.

13. Sai Saraswathi V, Saravanan D, Santhakumar K. Isolation of quercetin from the methanolic extract of Lagerstroemia speciosa by HPLC technique, its cytotoxicity against MCF-7 cells and photocatalytic activity. J Photochem Photobiol B Biol. 2017;171:20-6.

14. Yu YR, Rodriguez JR. Clinical presentation of Crohn's, ulcerative colitis, and indeterminate colitis: Symptoms, extraintestinal manifestations, and disease phenotypes. Semin Pediatr Surg. 2017; 26(6):349-55.

15. Ho GT, Porter RJ, Kalla R. Ulcerative colitis: Recent advances in the understanding of disease pathogenesis. F1000Research. 2020;9.

16. Kondo K, Hiramoto K, Yamate Y, Goto K, Sekijima H, Ooi K. Ameliorative effect of high-dose Vitamin $\mathrm{C}$ administration on dextran sulfate sodium-induced colitis mouse model. Biol Pharm Bull. 2019;42(6):954-9.

17. Yan $\mathrm{H}$, Wang $\mathrm{H}$, Zhang $\mathrm{X}$, Li $\mathrm{X}, \mathrm{Yu}$ J. Ascorbic acid ameliorates oxidative stress and inflammation in dextran sulfate sodiuminduced ulcerative colitis in mice. Int $\mathrm{J}$ Clin Exp Med. 2015;8(11):20245-53.

18. Jeon HJ, Yeom Y, Kim YS, Kim E, Shin $\mathrm{JH}$, Seok PR, et al. Effect of vitamin C on azoxymethane (AOM)/dextran sulfate sodium (DSS)-induced colitis-associated early colon cancer in mice. Nutr Res Pract. 2018;12(2):101-9.

19. Carr AC, Maggini S. Vitamin $C$ and immune function. Nutrients. 2017;9(11):125.

20. Mousavi S, Bereswill S, Heimesaat MM. Immunomodulatory and antimicrobial effects of vitamin C. Eur J Microbiol Immunol. 2019;9(3):73-9.

21. Priya TT, Sabu MC, Jolly CI. Free radical scavenging and anti-inflammatory properties of Lagerstroemia speciosa (L). Inflammopharmacology. 2008;16(4):182-7.

22. Zhang XY, Xu ZP, Wang W, Cao JB, Fu Q, Zhao WX, et al. Vitamin $C$ alleviates LPSinduced cognitive impairment in mice by suppressing neuroinflammation and oxidative stress. Int Immunopharmacol. 2018;65(February):438-47.

23. Rahmani J, Kord-Varkaneh H, Ryan PM, Rashvand S, Clark C, Day AS, et al. Dietary total antioxidant capacity and risk of ulcerative colitis: A case-control study. J Dig Dis. 2019;20(12):636-41.

How to cite this article: Sabilla Amrina Rosyada, Meddy Setiawan, Rahayu. Potential protection of vitamin $\mathrm{C}$ and Bungur leaf against ulcerative colitis. International Journal of Research and Review. 2022; 9(1): 623-629. DOI: https://doi.org/10.52403/ijrr.20220172 